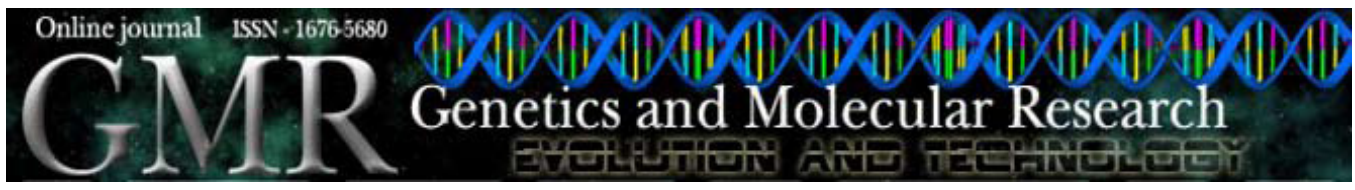

\title{
Genome-wide analysis of cyclins in maize (Zea mays)
}

\author{
X. Hu' ${ }^{1}$ X. Cheng ${ }^{2}$, H. Jiang ${ }^{2}$, S. Zhu ${ }^{2}$, B. Cheng ${ }^{2}$ and Y. Xiang ${ }^{1,2}$ \\ ${ }^{1}$ School of Forestry and Landscape Architecture, \\ Anhui Agricultural University, Hefei, Anhui, China \\ ${ }^{2}$ School of Life Science, Anhui Agricultural University, Hefei, Anhui, China \\ Corresponding author: Y. Xiang \\ E-mail: xiangyanahau@sina.com
}

Genet. Mol. Res. 9 (3): 1490-1503 (2010)

Received April 25, 2010

Accepted May 21, 2010

Published August 3, 2010

DOI 10.4238/vol9-3gmr861

\begin{abstract}
Cyclins are primary regulators of the activity of cyclindependent kinases and play crucial roles in cell cycle progression in eukaryotes. Although extensive studies have revealed the roles of some cyclins and underlying mechanisms in plants, relatively few cyclins have been functionally analyzed in maize. We identified 59 cyclins in the maize genome, distributed on 10 chromosomes; these were grouped into six types by phylogenetic analysis. The cyclin genes in the maize genome went through numerous tandem gene duplications on five chromosomes. However, no segmental duplications, which occur in rice, were found on maize chromosomes. This information allows us to assess the position of plant cyclin genes in terms of evolution and classification, which will be useful for functional studies of maize cyclins.
\end{abstract}

Key words: Cyclin; Gene duplication; Phylogenetic analysis; Zea mays 


\section{INTRODUCTION}

Similar to animals and yeasts, progression through the cell cycle is primarily driven by a family of cyclin-dependent kinases (CDKs) in plants. The catalytic activity of CDKs is directly regulated by binding and activation of cyclins, and can be further controlled by several additional mechanisms, including protein phosphorylation/dephosphorylation, proteolysis, and CDK inhibitor protein binding (Stals and Inze, 2001; Rossi and Varotto, 2002; Wang et al., 2004; La et al., 2006). Numerous studies have indicated that cyclins, as essential regulators of CDKs, not only activate CDKs by changing the conformation at their catalytic sites, but also contribute to the subcellular localization and substrate specificity of the complex as well as regulation of protein stability (Booher et al., 1989; Potuschak and Doerner, 2001; Wang et al., 2004).

Since the first discovery of cyclins in sea urchin eggs, at least 14 classes of cyclins have been discovered in animals, such as sea urchin, Caenorhabditis elegans, Drosophila melanogaster, and Homo sapiens (Nakamura et al., 1995; Pines, 2002; Wang et al., 2004). Plant cyclins were initially discovered in 1991 (Hata et al., 1991). Since then, more than 70 plant cyclins have been isolated from various plant species. In animals, multiple cyclins have been identified in several species, of which several have been described in the human genome. Mainly based on functions and sequence identity, the human cyclins involved in cell cycle control were classified into A- to I-type, K-, L-, T-type, and UNG2 (uracil DNA glycosylase 2) proteins (Nakamura et al., 1995; Pines, 2002). As in plants, these cyclins are classified as A-, B-, C-, D-, H-, and L-type cyclins (Renaudin et al., 1996; Yamaguchi et al., 2000; Barroco et al., 2003). There are 49 cyclins found in Arabidopsis, which are divided into 10 types on the basis of function and sequence analysis, including A- to D-type, H-, L-, T-, U-, SDS-, and J18-types (Wang et al., 2004). Similar to Arabidopsis, there are several common types between the two species, except C- and CycJ18-type, which are present in Arabidopsis but are absent in rice. However, F-type cyclins are only found in rice, and it has been suggested that this type is specific to monocotyledons (La et al., 2006). In maize, four D-type genes, Zeama;CycD2;1,Zeama;CycD4;1,Zeama;CycD4;2, and Zeama;CycD5;1 (Quiroz-Figueroa and Vázquez-Ramos, 2006), and five mitotic cyclins, Zeama;CycB1;1 (formerly cyclaZm), Zeama;CycB1;2 (formerly cyclbZm), Zeama;CycA1;1 (formerly cycIIZm), Zeama;CycB2;1 (formerly cycIIIZm), and Zeama;CycB1;3 (formerly CycZme1) (Renaudin et al., 1994; Sun et al., 1999), have been reported. Recently, whole genome sequences of maize became available (Schnable et al., 2009), providing an excellent opportunity to study plant cyclins extensively.

Previous studies indicated that a typical animal or plant cyclin contains a conserved 250-amino acid region called the cyclin core region, which consists of two domains: cyclin_N and cyclin_C (Nugent et al., 1991). The cyclin_N domain is also known as the cyclin box with a highly conserved sequence and is found in most of the known cyclins. It is about 100 amino acids long and contains the CDK-binding site. The cyclin_C domain is less conserved. Some known cyclins, such as the human cyclins G1 and G2, Arabidopsis C-, H-, T-, L-, J18-, P-, partial B- and D-type cyclins, have the cyclin_N domain but not the cyclin_C domain (Horne et al., 1996), suggesting that the cyclin_C domain may not be critical for its function. In general, cyclins are well conserved in both plants and animals. Some cyclins (A-, B- and D-type) have been studied in detail not only in yeast and animals, but also in plants. However, several types of animal cyclins, including the E-, G- and K-types, are not found in plants. D-type cyclins, also regarded as G1-specific cyclins because they are the first cyclins expressed in G1 phase, are conserved in animals and plants. They were proposed to be sensors of growth conditions and trigger the G1/S transition by activating the RBR/E2F-DP pathway (Gutierrez et al., 2002; Shen, 2002; Trimarchi and Lees, 2002). Further study revealed that plant D-type cyclins may 
function as mediators of internal and environmental stimuli to drive cell division. E-type cyclins are the next cyclins to be expressed in mid to late G1 phase and serve as important regulators in S-phase entry in animals; however, they lack homologues in plants (Obaya and Sedivy, 2002). Both B- and A-type cyclins in plants, known as mitotic cyclins, are expressed during mitosis. A-type cyclins are expressed soon after E-type cyclins at the G1/S boundary and form complexes with Cdk2. The activity of cyclin $\mathrm{A} / \mathrm{Cdk} 2$ is required for S-phase transition and control of DNA replication. B-type cyclins are expressed within a narrow time window from late $\mathrm{G} 2$ to mid $\mathrm{M}$ phase, and they contribute greatly to the G2/M transition (Lehner and O'Farrell, 1990; Pagano et al., 1992; Obaya and Sedivy, 2002). In previous studies, only a single A-type cyclin gene was found in invertebrates, and two were found in vertebrates (Nieduszynski et al., 2002). In contrast to animals, plants possess a higher complexity of A- and B-type cyclins, and much research has shown that they have extensive and complex functions. For example, Nicta; $C Y C A 3 ; 2$ has important functions, analogous to those of cyclin $\mathrm{E}$ in animals; the alfalfa A2-type cyclin Medsa;CYCA2;2 is expressed in all phases of the cell cycle (Roudier et al., 2000; Yu et al., 2003). In addition, some cyclins such as H-, L- and T-type, have been discovered in both animals and plants, and their functions have been studied in depth (Umeda et al., 1999; La et al., 2006).

In this study, 59 cyclin genes were identified in maize by database searches and were grouped into 6 types according to protein domains. Phylogenetic analyses were performed followed by the complete survey of genomic organization, as well as exon/intron and protein motif structural analyses of the cyclin family genes. On the basis of maize chromosome sequence clone information, these cyclin genes were mapped to 10 different maize chromosomes. Furthermore, tandem gene and segmental duplication events that occurred on maize chromosomes were investigated. The results of these group classifications and investigation of gene duplication will be useful for future studies of cyclin families.

\section{MATERIAL AND METHODS}

\section{Identification of putative maize cyclins}

To retrieve data on maize cyclins, a series of searches in NCBI were performed. Maize protein sequences were downloaded from the website $h$ ttp://www.maizesequence.org/index.html, and then Hidden Markov Model (HMM) profiles of cyclin_N domains and cyclin_C domains from rice were obtained using the Pfam database (http://pfam.janelia.org/search/sequence). As cyclin_N domains are found in all known cyclins and are highly conserved, we used the BLASTP program to search the maize protein database with the HMM profile of cyclin_N domain as a query. The e-value chosen in BLASTP was set at le-3. Finally, overlapping genes were removed using a complete sequence alignment method in MEGA 3.1 to obtain the final set of non-overlapping set of cyclin genes in maize.

\section{Nomenclature of the predicted maize cyclin genes}

We consulted the principles adopted in other articles (Vandepoele et al., 2002; Wang et al., 2004; Jiang and Ramachandran, 2004) and the nomenclature put forward by Renaudin et al. (1996). The newly identified genes in this study were named in line with the nomenclature used for the previously identified genes and on the basis of their phylogenetic relatedness to other members of the same family. Because both maize and rice are monocotyledons, the nomenclature of the predicted maize cyclin genes was mainly based on the phylogenetic relationship of the protein sequences between rice and maize. 


\section{Phylogenetic analysis of cyclin genes in maize}

The phylogenetic tree including maize and rice cyclin genes was generated by MEGA 3.1 as well as the phylogenetic tree including Arabidopsis and maize with the following parameters: Poisson correction, pairwise deletion, bootstrap (1000 replicates) (Kumar et al., 2004).

\section{Conserved motif analysis}

To identify the conserved motifs in cyclin proteins, the protein sequences of maize cyclins were merged using the GENESTUDIO software, and then the protein sequences of maize cyclin genes were analyzed using MEME (Multiple EM for Motif Elicitation).

\section{Mapping cyclins on maize chromosomes}

In this study, we used the DNA TOOLS software to set up local databases of the maize complete nucleotide sequences, and the starting position of all cyclin genes on each chromosome was obtained using tBLASTn $(\mathrm{P}=0.001)$. Using this method, the physical locations of all cyclin genes were confirmed. Finally, the chromosome map showing the physical location of all cyclin genes was generated using the Genome Pixelizer software (http://www.niblrrs. ucdavis.edu/GenomePixelizer/GenomePixelizer_Welcome.html).

\section{Discovery of duplications of maize cyclin genes}

In an attempt to detect tandem and segmental duplications of cyclin genes throughout the maize genome, we utilized a modified method described previously to find duplicated blocks (Vision et al., 2000; Vandepoele et al., 2002). First, the local protein databases that consisted of 59 putative maize cyclin protein sequences were constructed with DNA TOOLS, and homologues with high sequence similarities were found by BLASTP search. We then used the highly similar sequences as query to search the maize bacterial artificial chromosome (BAC) database to determine the number and length of the exons. Finally, tandem and segmental duplications were confirmed by sequence alignment, exon comparisons and chromosome location analysis.

\section{RESULTS}

\section{Identification of cyclins}

Using the BLAST program available from the Pfam database, the total predicted 79 gene models were confirmed as cyclin genes in maize. After removing the overlapping genes, a total of 59 predicted protein sequences were eventually confirmed as cyclin genes in maize. The overall analysis of the complete genome of maize revealed that the maize cyclin gene family comprises 59 genes (see Material and Methods). All predicted genes and related information are listed in Table 1 including their chromosome locations, sequenced ID, genetic map positions, and number of encoded amino acids. The molecular weights of these deduced cyclin protein sequences generally range from $17.2(\mathrm{CycD} 4 ; 9)$ to $111.7 \mathrm{kDa}(\mathrm{CycB} 1 ; 7)$. 
Table 1. Predicted genes and related information including chromosome locations, sequenced ID, genetic map positions, and number of encoded amino acids.

\begin{tabular}{|c|c|c|c|c|c|c|}
\hline Cyclin name & Sequenced ID & Chromosome & Position (Mb) & Number of AA & MW (kDa) & PI \\
\hline Zeama;CycA1;1 & GRMZM2G017081_P01 & 5 & 59.1 & 489 & 55.2 & 9.11 \\
\hline Zeama;CycA2;1 & GRMZM2G059922_P01 & 10 & 17.6 & 485 & 53.7 & 8.36 \\
\hline Zeama;CycA2;2 & GRMZM2G059922_P02 & 10 & 17.6 & 404 & 44.3 & 8.88 \\
\hline Zeama;CycA3;1 & GRMZM2G026346_P01 & 1 & 254.9 & 305 & 33.8 & 8.33 \\
\hline Zeama;CycA3;2 & GRMZM2G026346_P02 & 1 & 254.9 & 372 & 41.4 & 9.09 \\
\hline Zeama;CycA3;3 & GRMZM2G363437_P01 & 5 & 18.5 & 357 & 40.3 & 8.51 \\
\hline Zeama;CycA3;4 & GRMZM2G363437_P02 & 5 & 18.5 & 351 & 39.7 & 8.73 \\
\hline Zeama;CycA3;5 & GRMZM2G060690_P01 & 1 & 161.5 & 382 & 41.6 & 8.92 \\
\hline Zeama;CycA3;6 & GRMZM2G060690_P03 & 1 & 161.5 & 423 & 46.1 & 8.85 \\
\hline Zeama;CycA3;7 & GRMZM2G180728 P01 & 3 & 108.4 & 433 & 46.9 & 8.30 \\
\hline Zeama;CycA3;8 & GRMZM2G180728_P02 & 3 & 108.4 & 474 & 51.6 & 8.67 \\
\hline Zeama;CycB1;1 & GRMZM2G310115 P04 & 8 & 170.1 & 449 & 50.0 & 9.19 \\
\hline Zeama;CycB1;2 & GRMZM2G034647_P01 & 8 & 118.7 & 446 & 48.3 & 9.49 \\
\hline Zeama;CycB1;3 & GRMZM2G005619_P01 & 8 & 1.5 & 479 & 53.3 & 9.22 \\
\hline Zeama;CycB1;4 & GRMZM2G025200_P01 & 4 & 15.0 & 601 & 67.7 & 8.63 \\
\hline Zeama;CycB1;5 & GRMZM2G073003 P03 & 3 & 185.8 & 228 & 26.2 & 6.00 \\
\hline Zeama;CycB1;6 & GRMZM2G073003_P01 & 3 & 185.8 & 442 & 49.5 & 9.14 \\
\hline Zeama;CycB1;7 & GRMZM2G310115_P02 & 8 & 170.1 & 1003 & 111.7 & 8.47 \\
\hline Zeama;CycB2;1 & GRMZM2G073671_P01 & 6 & 81.5 & 424 & 47.7 & 5.75 \\
\hline Zeama;CycB2;2 & GRMZM2G138886_P01 & 2 & 17.4 & 424 & 47.5 & 5.50 \\
\hline Zeama;CycB2;3 & GRMZM2G073671_P01 & 6 & 81.5 & 424 & 47.7 & 5.75 \\
\hline Zeama;CycD1;1 & GRMZM2G476685P01 & 7 & 99.9 & 325 & 34.9 & 4.72 \\
\hline Zeama;CycD2;1 & GRMZM2G075117 P02 & 4 & 178.7 & 360 & 38.9 & 5.46 \\
\hline Zeama;CycD2;2 & GRMZM2G079629 P01 & 7 & 160.5 & 345 & 37.8 & 5.65 \\
\hline Zeama;CycD3;1 & GRMZM2G161382_P01 & 2 & 160.5 & 270 & 28.8 & 4.67 \\
\hline Zeama;CycD3;2 & GRMZM2G161382_P02 & 2 & 160.5 & 391 & 40.6 & 4.82 \\
\hline Zeama;CycD4;1 & GRMZM2G133413 P01 & 1 & 200.6 & 388 & 41.9 & 5.44 \\
\hline Zeama;CycD4;2 & GRMZM2G133413_P02 & 1 & 200.6 & 253 & 26.6 & 7.50 \\
\hline Zeama;CycD4;3 & GRMZM2G133413_P03 & 1 & 200.6 & 187 & 20.8 & 9.78 \\
\hline Zeama;CycD4;4 & GRMZM2G178229-P01 & 2 & 184.5 & 361 & 39.1 & 5.64 \\
\hline Zeama;CycD4;5 & GRMZM2G178229_P02 & 2 & 184.5 & 291 & 32.2 & 5.60 \\
\hline Zeama;CycD4;6 & GRMZM2G178229-P03 & 2 & 184.5 & 290 & 31.7 & 7.09 \\
\hline Zeama;CycD4;7 & GRMZM2G140633 P01 & 7 & 122.1 & 356 & 38.8 & 5.63 \\
\hline Zeama;CycD4;8 & GRMZM2G140633_P02 & 7 & 122.1 & 274 & 30.3 & 5.37 \\
\hline Zeama;CycD4;9 & GRMZM2G140633_P03 & 7 & 122.1 & 158 & 17.2 & 4.27 \\
\hline Zeama;CycD $4 ; 10$ & GRMZM2G140633_P05 & 7 & 122.1 & 348 & 38.0 & 5.91 \\
\hline Zeama;CycD5;1 & GRMZM2G006721 P02 & 9 & 143.4 & 354 & 37.7 & 5.41 \\
\hline Zeama;CycD5;2 & GRMZM2G007130_P01 & 1 & 22.4 & 345 & 37.3 & 5.23 \\
\hline Zeama;CycD5;3 & GRMZM2G047637 P01 & 3 & 109.6 & 349 & 37.9 & 4.75 \\
\hline Zeama;CycD5;4 & GRMZM2G056303_P01 & 1 & 253.8 & 349 & 36.9 & 4.83 \\
\hline Zeama;CycD6;1 & GRMZM2G050933_P01 & 7 & 151.0 & 307 & 33.0 & 5.70 \\
\hline Zeama;CycD7;1 & GRMZM2G058410_P01 & 4 & 0.8 & 431 & 47.4 & 5.66 \\
\hline Zeama;CycF2;1 & GRMZM2G001164_P01 & 5 & 181.8 & 382 & 43.3 & 7.67 \\
\hline Zeama;CycF $2 ; 2$ & GRMZM2G401680-P01 & 5 & 173.9 & 244 & 27.0 & 5.13 \\
\hline Zeama;CycF $2 ; 3$ & AC212449.4_FGP004 & 5 & 174.1 & 284 & 31.3 & 5.61 \\
\hline Zeama;CycF $2 ; 4$ & AC212449.4_FGP001 & 5 & 174.1 & 400 & 43.0 & 5.69 \\
\hline Zeama;CycF $2 ; 5$ & AC212449.4_FGP002 & 5 & 174.1 & 343 & 38.0 & 5.66 \\
\hline Zeama;CycF2;6 & AC212449.4 FGP006 & 5 & 174.0 & 343 & 38.0 & 5.46 \\
\hline Zeama;SDS-1 & GRMZM2G093157_P01 & 9 & 141.1 & 455 & 51.0 & 5.09 \\
\hline Zeama;SDS-2 & GRMZM2G093157 P02 & 9 & 141.1 & 459 & 51.4 & 5.09 \\
\hline Zeama;SDS-3 & GRMZM2G344416 P01 & 1 & 28.8 & 651 & 72.6 & 4.99 \\
\hline Zeama;SDS-4 & GRMZM2G378422_P01 & 2 & 102.7 & 327 & 36.3 & 4.74 \\
\hline Zeama;CycT1;1 & GRMZM2G068340_P01 & 5 & 188.7 & 605 & 67.8 & 5.18 \\
\hline Zeama;CycT1;2 & GRMZM2G101635 P01 & 4 & 147.8 & 608 & 68.2 & 5.19 \\
\hline Zeama;CycT1;3 & GRMZM2G462118_P01 & 5 & 75.2 & 389 & 43.8 & 9.32 \\
\hline Zeama;CycTl;4 & AC233979.1_FGP009 & 10 & 134.8 & 487 & 56.1 & 7.05 \\
\hline Zeama;CycT1;5 & GRMZM2G308570_P02 & 1 & 3.9 & 493 & 56.7 & 6.69 \\
\hline Zeama;CycT1;6 & GRMZM2G115670_P01 & 7 & 82.5 & 631 & 71.1 & 6.18 \\
\hline Zeama;CycTl;7 & GRMZM2G377311_P01 & 1 & 13.3 & 592 & 66.2 & 6.87 \\
\hline
\end{tabular}

Generic name: designated in previous publications (La et al., 2006). AA = amino acids; $\mathrm{MW}=$ molecular weight; $\mathrm{PI}=$ isoelectric point of the deduced polypeptide. 
To study the evolutionary relationships of cyclin proteins and to classify the newly uncovered members in maize, a phylogenetic tree was generated using 106 putative cyclin sequences, including 59 genes from maize and 47 genes from rice (La et al., 2006) (Figure 1). The maize and rice cyclin genes clustered into nine groups (A, B, D, F, H, L, P, T, and SDS) similar to the phylogenetic tree including rice and Arabidopsis (La et al., 2006). There are several common types between the two species, not including the H-, L- and P-types, which are present in rice but not in maize. In the cyclin A group, the types of eight maize genes are closely related to two rice genes, CYA3;1,CYA3;2, while the remaining four maize genes are closely related to four rice genes including CYA2;1, CYA1;1, CYA1;2, and CYA1;4. These genes were designated cycA1;1-cycA1;8, cycA2;1, cycA2;2, and cycA1;1 on the basis of high sequence similarity to A-type cyclins in rice. Interestingly, two F-type cyclins, CYF3;1 and CYF3;2, in maize are closely related to A-type cyclins belonging to maize and rice. The Fgroup contains six maize genes and six rice genes, including the CYF2 and CYF1 subgroups, and the members from maize were designated cycF2;1-cycF2;6 on the basis of sequence similarity with the corresponding F-type genes in rice. Similarly, the nomenclature of other maize cyclin genes, including the B-, D-, T-, and SDS-types, were also confirmed on the basis of the phylogenetic relationship of the rice genes with the corresponding maize cyclin genes. As a result, all maize cyclins were grouped into A-, B-, D-, F-, SDS-, and T-types, and the numbers of genes in these types were $11,10,21,6,4$, and 7, respectively.

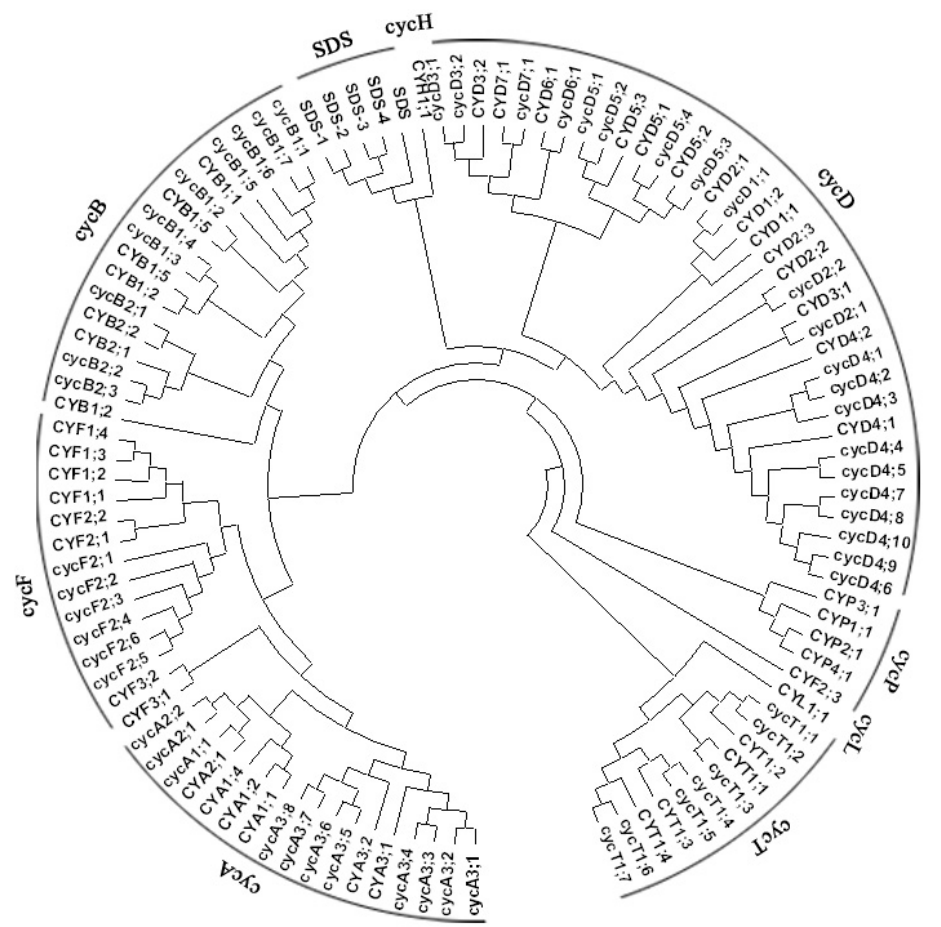

Figure 1. Unrooted neighbor joining phylogenetic tree of the maize and rice cyclins. The cyclins with "cyc" designations and SDS-1-SDS-4 are from maize. The remaining cyclins are from rice. There are six types of cyclins shared between rice and maize, whereas rice has the H-, L-, and P-type cyclins, which are not found in maize. 


\section{Phylogenetic analysis of predicted maize cyclins}

To further study the evolutionary relationships between the genes in the maize cyclin family, a phylogenetic tree was constructed based on maize cyclin_N domain sequences. As shown in Figure 2, the 59 maize cyclin proteins can be divided into 6 types. Each type of cyclin formed a relatively separate clade, and the relationships among these clades are also generally consistent with that in Figure 1. A- and B-type maize cyclins are more closely related compared with other types. D-type members form the largest cluster in the maize cyclin family, which is closer to the A-, B-, F-, and SDS-type clades. T-type cyclins form a separate clade, making them more independent in the tree. In addition, Figure 2 presents the domain structure with the corresponding cyclin genes in maize. All B- and F-type cyclins contain cyclin_N and cyclin_C domains; 91\% A-type, 75\% SDS-type and 48\% D-type members contain both domains. However, all T-type cyclins have only cyclin_N domains in maize.

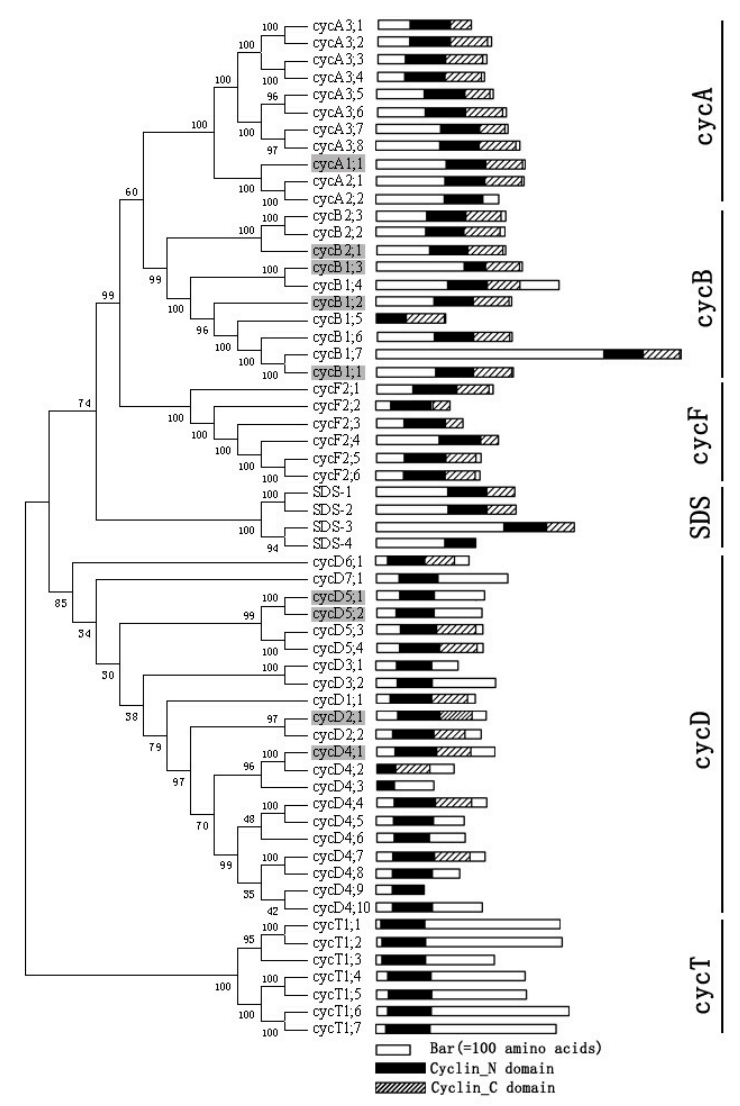

Figure 2. Phylogenetic tree and domain structure of the 59 predicted maize cyclins. The numbers at the branches represent bootstrap values. All the B- and F-type cyclins possess both cyclin_N and cyclin_C domains; most of the A- and SDS-type cyclins also have both domains. However, each of the T-type cyclins has only the cyclin_N domain without a cyclin_C domain. Those cyclins highlighted in gray boxes have been described in previous studies. 


\section{Analysis of conserved motifs in maize}

Conserved motifs were investigated by MEME, and 15 conserved motifs were detected (Figure 3). Among these 15 conserved motifs, 5 motifs (1, 2, 3, 8, and 9) are located within the cyclin-box domain. In fact, the conserved motifs encoding the cyclin_N domain can be found in all 59 cyclin genes of maize, while the cyclin_C domain is only found in some cyclins. This result confirmed that the $\mathrm{C}$ domain is a less conserved region than the $\mathrm{N}$ domain in plants.
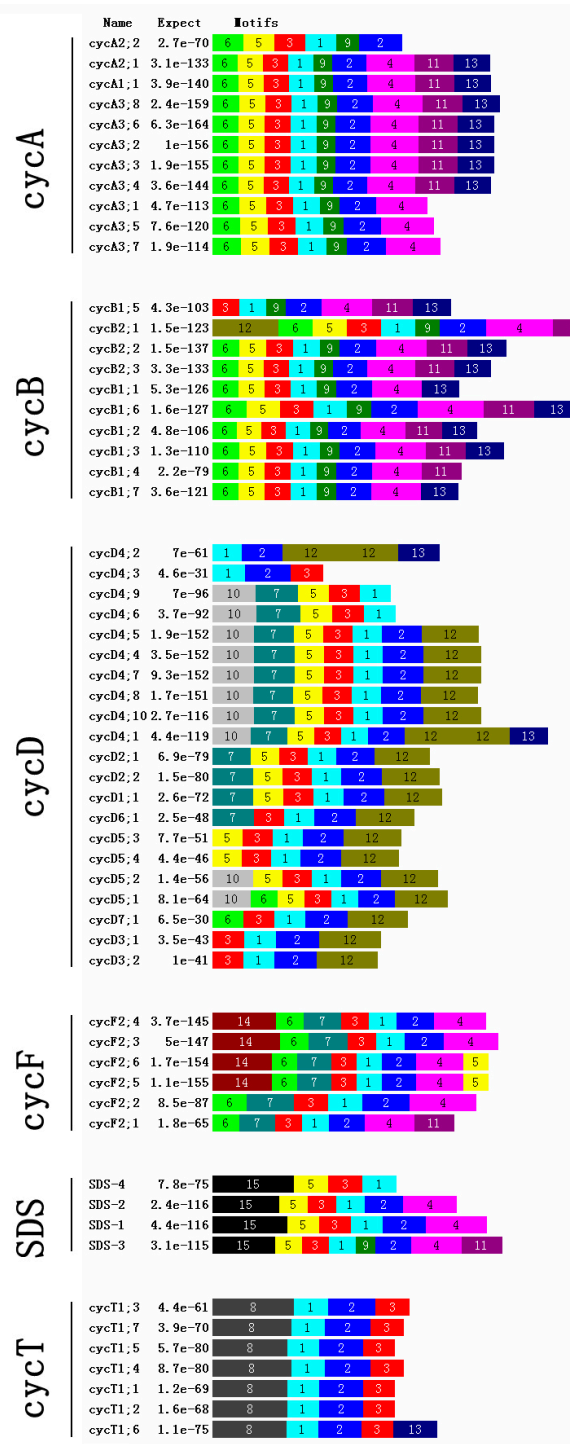

cyclin_N:motif $1,2,3$ and 9

cyclin_N:motif $1,2,3$ and 9

cyclin_N:motif 1,2 and 3

cyclin_N:motif 1,2 and 3

cyclin_N:motif $1,2,3$ and 9

cyclin_N:motif $1,2,3$ and 8

Figure 3. Fifteen putative conserved motifs were identified in the cyclin family in maize using the MEME search tool. Different motifs are indicated by different colors, but motif sizes are not related to the length of box. 
In A- and B-type cyclin genes, four conserved motifs (1, 2, 3, and 9) are found in all cyclins, suggesting that the phylogenetic relationship of these two types of cyclin genes is close. Most of the D-type genes (except cycD4;2, cycD4;6 and cycD4;9) contain three motifs including 1, 2 and 3, and were similar to the F-type genes. Among the T-type genes, all members contain motifs $1,2,3$, and 8 .

\section{Distribution of predicted cyclin genes on maize chromosomes}

For the distribution of predicted cyclin genes on maize chromosomes, searches were performed for map positions and genetic distances of corresponding cyclin genes against maize protein databases. The 59 cyclin family genes are distributed on 10 maize chromosomes; however, no cyclin gene is located on chromosome 0 (Figure 4). In maize, the highest number of cyclin genes is located on chromosome 1 (12 genes), the longest chromosome, whereas only one cyclin gene, cycB2;1, is found on chromosome 6 . The distribution of cyclin genes on other chromosomes is also uneven: eleven genes on chromosome 5 , eight genes on chromosome 7 , seven genes on chromosome 2 , five genes on chromosome 3 , four genes on chromosome 4, 8, 10, and three genes on chromosome 9 .

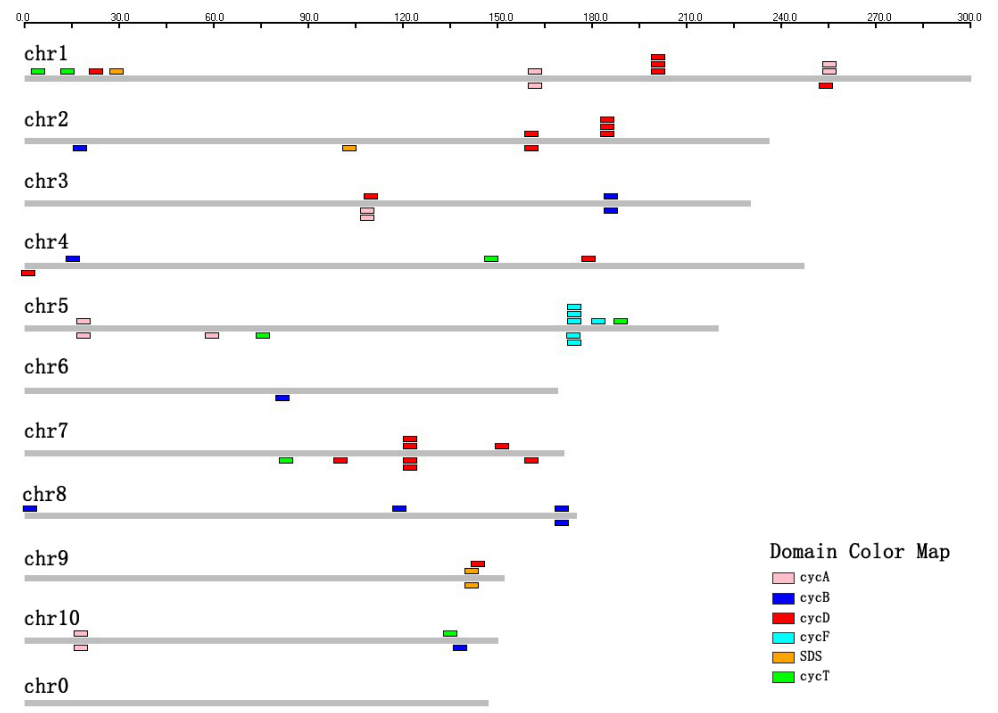

Figure 4. Physical locations of maize cyclin genes. The boxes above and below the chromosomes (chr; represented as gray bars) designate the approximate locations of the 11 maize cyclin genes.

Each type of cyclin gene except the F-type is found on at least three chromosomes, and B- and D-type cyclins are dispersed on up to six chromosomes. Interestingly, all F-type cyclin genes are exclusively located on chromosome 5 , and 4 of them constitute a gene cluster with high sequence similarity. We also observed that gene clusters are distributed among all chromosomes except chromosomes 0,4 , and 6 . Among the 13 clusters found on maize chromosomes, 3 are located on chromosome 1, 2 each on chromosomes 2, 3 and 5, and only 1 cluster on each of chromosomes 7, 8, 9, and 10 . 


\section{Genome organization of predicted cyclin genes in maize}

In an attempt to study whether gene tandem or segmental duplications led to the acquisition of new cyclin genes in maize, we performed a series of BLASTN searches, sequence alignment and location comparisons on chromosomes as well as comparisons of exon numbers and lengths. We discovered seven tandem duplication events, which occurred on chromosomes $1,2,3,5$, and 7 . However, no segmental duplications were found on maize chromosomes.

As shown in Figure 5A, CycA3;1 and CycA3;2 genes are located on the same BAC clone on chromosome 1 and constitute a gene cluster; the identity of their amino acids is $81.9 \%$. Even in $\mathrm{CycA} 3 ; 1$ and $\mathrm{CycA} 3 ; 2$, the exon numbers are 5 and 7, respectively, and the lengths of five exons in CycA3;1 are identical to that of the corresponding exons in CycA3;2. Moreover, the exons marked with the same signs in Figure 5 share the same nucleotide sequences. According to these observations, we presumed that one of these genes, CycA3;1 or CycA3;2, was derived from the other gene and formed a tandem gene duplication. Six groups altogether, CycA3;7 and CycA3;8, CycD3;1 and CycD3;2, CycD4;7 and CycD4;8, CycD4;9 and CycD4;10, CycF2;3 and $\mathrm{CycF} 2 ; 4, \mathrm{CycF} 2 ; 5$ and $\mathrm{CycF} 2 ; 6$, formed tandem gene duplications (Figure 5B).
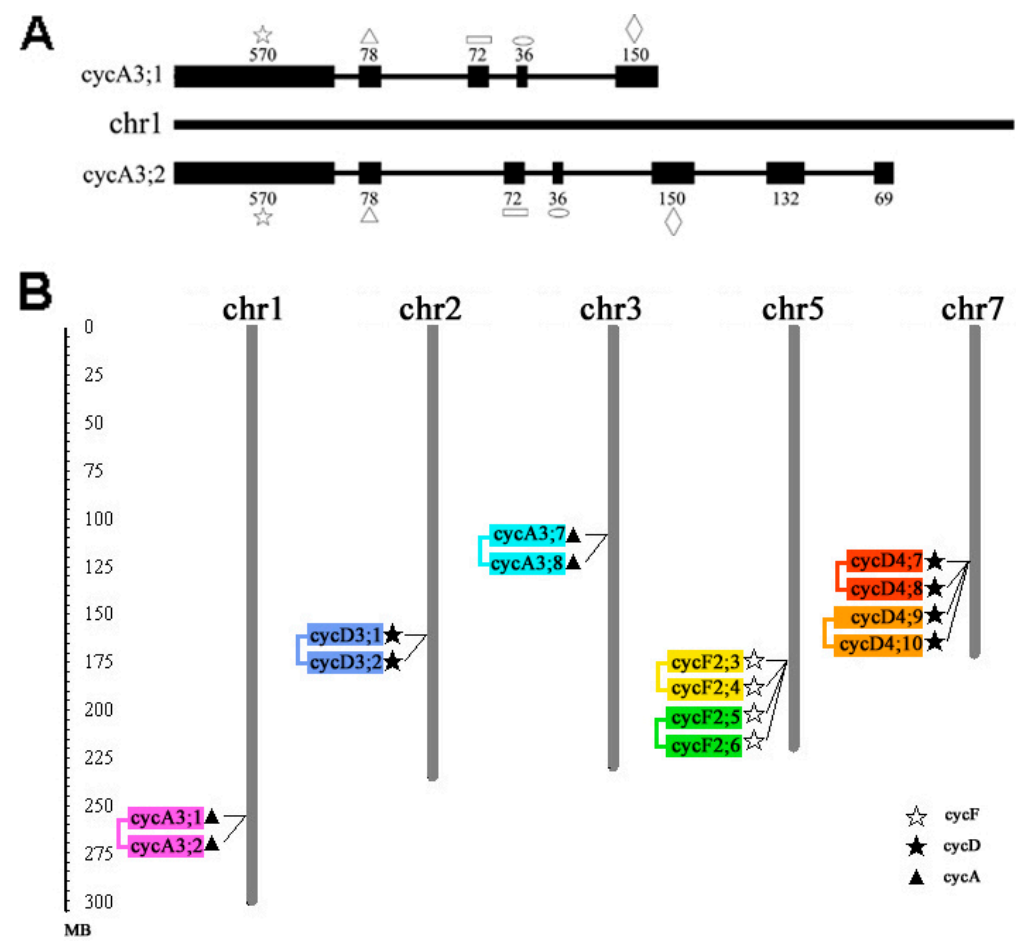

Figure 5. A. Gene tandem duplication of two A-type cyclins. The black bars represent exons, and spaces between two bars represent introns. The numbers above the exons refer to the number of nucleotides. Those exons marked with the same signs share the same nucleotide sequences. B. Distribution of cyclin gene duplications on 5 maize chromosomes. Gene tandem duplications are highlighted with different colors. The colored bands connect corresponding tandem duplicated genes. 


\section{DISCUSSION}

Cyclin proteins are components of the regulatory system that controls the orderly progression of the events of cell division in eukaryotes. Pines et al. (2002) found and analyzed at least 22 cyclins in the human genome. In the C. elegans genome, 34 cyclins have been found (Plowman et al., 1999). In higher plants, the number of cyclins vary from 49 in Arabidopsis to 48 in rice, suggesting that higher plant genomes contain greater numbers of the cyclin genes than those of animals. In our study, 59 predicted cyclins were identified in maize, which contains more members compared with Arabidopsis and rice. A reasonable explanation for these facts is that the plant cell cycle has unique features compared to the animal cell cycle, including the need to form unique cytoskeletal structures called preprophase band and phragmoplast, the formation of diffuse mitotic spindles, and distinctive cytokinesis (Cyr and Palevitz, 1995; Smith, 1999; Breyne et al., 2002). We also presumed that the cell cycle genes in higher plants, especially in maize, may be integrated into a more complex system, and plant-specific regulatory pathways of cell division may exist.

In this study, we identified and analyzed a large number of maize sequences by using the conserved cyclin_N domain from rice (La et al., 2006) as query sequences. A total of 59 cyclin genes in the maize genome were identified, and they belong to the A, B, D, F, T, and SDS families. D-type cyclins perceive the cell's environment, acting as growth sensors to promote initiation and/or establishment of the G1 phase (Sherr and Roberts, 1999). As previously described (Quiroz-Figueroa and Vázquez-Ramos, 2006), CycD4;1 is poorly represented in germinating seeds but highly represented in other proliferating or non-proliferating tissues. CycD5;1 is the least expressed, but has the highest expression in leaf tissue. D-type cyclins contain the highest number (21) of cyclin genes, approximately half of the cyclins in maize, suggesting that there may be many D-type cyclins involved in the G1 phase, and some of these cyclins may be tissue-specific in maize.

Phylogenetic analysis indicated that there are 9 and 6 classes of cyclins in rice and maize genomes, respectively. Six types (A-, B-, D-, F-, T-, and SDS-types) are shared by the two species, whereas rice has the H-, L-, and P-type cyclins that are not found in maize. As shown in Figure 1, the same types of cyclins in rice and maize are located in the same clades, and they share high sequence similarities. By contrast, the H-, L-, and P-types in rice form a separate clade. It is interesting to note, however, that most of the F-type cyclins are grouped together, and one (CYF2;3) that is apart from the other F-type cyclins happens to be closely related to P-type cyclins in rice.

We also studied the evolutionary relationships between maize and Arabidopsis, and the results show that Arabidopsis and maize share five types of cyclins, A-, B-, D-, T-, and SDS-types, whereas C-, H-, L-, U-, and J18-type cyclins are specific to Arabidopsis. However, F-type cyclins are not found in Arabidopsis. Compared with humans and animals, plants contain fewer cyclin types, especially maize, which only possesses six types of cyclin genes. Nevertheless, the number of genes in each cyclin type in plants is much more than that of the corresponding type in humans and animals. In humans, none of the cyclin types contains more than three members; however, half of cyclin types in maize possess at least ten cyclins, including 11 A-type, 10 B-type and 21 D-type cyclins. All of these facts suggest that some of the A- B- and D-types may assume the functions that are carried out by the animal-specific cyclins. For example, plants lack E-type cyclins, which are known as very important regulators involved in $\mathrm{G1} / \mathrm{S}$ checkpoint control in animals. It was 
recently reported that Nicta;CycA3;2 acts as an early G1/S-activated gene with functions that are analogous to those of cyclin $\mathrm{E}$ in animals (Yu et al., 2003). Even among the plant species analyzed so far, the classes of cyclins vary significantly. Maize lacks H-, L- and P-type cyclins, but possesses additional F-type cyclins compared with Arabidopsis, which may function as H-, L-, and P-type cyclins. In addition, rice and maize share F-type cyclins, while Arabidopsis lacks the corresponding type. Previous analysis of the cyclin gene family in rice identified the F-type as a new type of cyclin with no corresponding homologues found in other plant species. Our study reveals that maize is the second plant in which F-type cyclins have been identified. Thus, it is likely that this type of cyclin may be unique to monocotyledons. All the results above confirm that cyclins play complicated and diverse roles in cell cycle progression in plants. Therefore, it is important to analyze the full collection of functional cyclin genes in order to fully understand plant cyclin diversity (John et al., 2001).

We further analyzed the conserved motifs in maize cyclin family by MEME. The majority of cyclin genes in the same type have similar motifs, suggesting that these conserved motifs play crucial roles in subfamily-specific functions. However, SDS-type proteins are very divergent in their structures. For example, cycSDS- 4 contains only motifs 1 and 3 in its cyclin_N region, whereas cycSDS-3 has motifs 1, 2, 3, and 9, suggesting the complex nature of the function of SDS-type cyclins in maize. It was interesting that the cyclin_N domain in T-type cyclins possesses a specific motif (motif 8), which was not found in other type of maize cyclins. A possible explanation is that motif 8 may have a specific function, and that T-type cyclins play specific roles in cell cycle progression.

The analysis of the chromosomal distribution of cyclin genes in maize shows that great differences exist in their distribution. There are 12 cyclin genes located on chromosome 1 , which possesses the highest number of cyclin genes, but no cyclin gene is found on chromosome 0 . Unlike other cyclin types dispersed throughout the whole genome, all members in F-type cyclins are exclusively located on chromosome 5, which is consistent with the distribution of corresponding homologues in rice (La et al., 2006). According to Vandepoele et al. (2002), studies in rice show that cyclins in Arabidopsis experienced large-scale duplications, including segmental and tandem duplications. La et al. (2006) found that there are four Dtype, two each of A-type and D-type, and one each of B-type and T-type cyclins organized in segmental or tandem duplications. Similarly, we also found several tandem duplication events located in the maize chromosome. All the results above suggest that the increased number of cyclin genes correlates closely with the frequency of duplication events. It is worth noting that the tandem duplicated genes in maize also formed gene clusters. For example, CycA3;1 and $\mathrm{CycA} 3 ; 2$ not only formed tandem duplications, but also constituted a gene cluster on chromosome 1. Therefore, we speculate that in maize, tandem duplications may be the basis for the formation of gene clusters. In addition, no segmental duplications were found in the maize chromosomes, unlike in rice and Arabidopsis. This analysis reveals that there are potentially more duplication events in rice and Arabidopsis genome compared with maize.

\section{ACKNOWLEDGMENTS}

Research supported by grants from the National Natural Science Foundation of China (\#10675002) and National High-Tech Research and Development Program (863 Program) (\#2006AA10Z1B4). We thank members of the Key Lab of Crop Biotechnology of Anhui province and Xiao Cheng for assistance in conducting these experiments. 


\section{REFERENCES}

Barroco RM, De Veylder L, Magyar Z, Engler G, et al. (2003). Novel complexes of cyclin-dependent kinases and a cyclinlike protein from Arabidopsis thaliana with a function unrelated to cell division. Cell Mol. Life Sci. 60: 401-412.

Booher RN, Alfa CE, Hyams JS and Beach DH (1989). The fission yeast cdc2/cdc13/suc1 protein kinase: regulation of catalytic activity and nuclear localization. Cell 58: 485-497.

Breyne P, Dreesen R, Vandepoele K, De Veylder L, et al. (2002). Transcriptome analysis during cell division in plants. Proc. Natl. Acad. Sci. U.S.A. 99: 14825-14830.

Cyr RJ and Palevitz BA (1995). Organization of cortical microtubules in plant cells. Curr. Opin. Cell Biol. 7: 65-71.

Gutierrez C, Ramirez-Parra E, Castellano MM and del Pozo JC (2002). G(1) to S transition: more than a cell cycle engine switch. Curr. Opin. Plant Biol. 5: 480-486.

Hata S, Kouchi H, Suzuka I and Ishii T (1991). Isolation and characterization of cDNA clones for plant cyclins. EMBO J. 10: 2681-2688.

Horne MC, Goolsby GL, Donaldson KL, Tran D, et al. (1996). Cyclin G1 and cyclin G2 comprise a new family of cyclins with contrasting tissue-specific and cell cycle-regulated expression. J. Biol. Chem. 271: 6050-6061.

Jiang S and Ramachandran S (2004). Identification and molecular characterization of myosin gene family in Oryza sativa genome. Plant Cell Physiol. 45: 590-599.

John PCL, Mews M and Moore R (2001). Cyclin/CDK complexes: Their involvement in cell cycle progression and mitotic division. Protoplasma 216: 119-142.

Kumar S, Tamura K and Nei M (2004). MEGA3: Integrated software for molecular evolutionary genetics analysis and sequence alignment. Brief Bioinform. 5: 150-163.

La H, Li J, Ji Z, Cheng Y, et al. (2006). Genome-wide analysis of cyclin family in rice (Oryza sativa L.). Mol. Genet. Genomics 275: 374-386.

Lehner CF and O'Farrell PH (1990). The roles of Drosophila cyclins A and B in mitotic control. Cell 61: 535-547.

Nakamura T, Sanokawa R, Sasaki YF, Ayusawa D, et al. (1995). Cyclin I: a new cyclin encoded by a gene isolated from human brain. Exp. Cell Res. 221: 534-542.

Nieduszynski CA, Murray J and Carrington M (2002). Whole-genome analysis of animal A- and B-type cyclins. Genome Biol. 3: RESEARCH0070.

Nugent JH, Alfa CE, Young T and Hyams JS (1991). Conserved structural motifs in cyclins identified by sequence analysis. J. Cell Sci. 99 (Pt 3): 669-674.

Obaya AJ and Sedivy JM (2002). Regulation of cyclin-Cdk activity in mammalian cells. Cell Mol. Life Sci. 59: 126-142.

Pagano M, Pepperkok R, Verde F, Ansorge W, et al. (1992). Cyclin A is required at two points in the human cell cycle. EMBO J. 11: 961-971.

Pines J (2002). Confirmational change. Nature 376: 294-295.

Plowman GD, Sudarsanam S, Bingham J, Whyte D, et al. (1999). The protein kinases of Caenorhabditis elegans: a model for signal transduction in multicellular organisms. Proc. Natl. Acad. Sci. U.S.A. 96: 13603-13610.

Potuschak T and Doerner P (2001). Cell cycle controls: genome-wide analysis in Arabidopsis. Curr. Opin. Plant Biol. 4: 501-506.

Quiroz-Figueroa F and Vázquez-Ramos JM (2006). Expression of maize D-type cyclins: comparison, regulation by phytohormones during seed germination and description of a new D cyclin. Physiol. Plantarum 128: 556-568.

Renaudin JP, Colasanti J, Rime H, Yuan Z, et al. (1994). Cloning of four cyclins from maize indicates that higher plants have three structurally distinct groups of mitotic cyclins. Proc. Natl. Acad. Sci. U.S.A. 91: 7375-7379.

Renaudin JP, Doonan JH, Freeman D, Hashimoto J, et al. (1996). Plant cyclins: a unified nomenclature for plant A-, B- and D-type cyclins based on sequence organization. Plant Mol. Biol. 32: 1003-1018.

Rossi V and Varotto S (2002). Insights into the G1/S transition in plants. Planta 215: 345-356.

Roudier F, Fedorova E, Gyorgyey J, Feher A, et al. (2000). Cell cycle function of a Medicago sativa A2-type cyclin interacting with a PSTAIRE-type cyclin-dependent kinase and a retinoblastoma protein. Plant J. 23: 73-83.

Schnable PS, Ware D, Fulton RS, Stein JC, et al. (2009). The B73 maize genome: complexity, diversity, and dynamics. Science 326: 1112-1115.

Shen WH (2002). The plant E2F-Rb pathway and epigenetic control. Trends Plant Sci. 7: 505-511.

Sherr CJ and Roberts JM (1999). CDK inhibitors: positive and negative regulators of G1-phase progression. Genes Dev. 13: 1501-1512.

Smith LG (1999). Divide and conquer: cytokinesis in plant cells. Curr. Opin. Plant Biol. 2: 447-453.

Stals H and Inze D (2001). When plant cells decide to divide. Trends Plant Sci. 6: 359-364.

Sun Y, Flannigan BA and Setter TL (1999). Regulation of endoreduplication in maize (Zea mays L.) endosperm. Isolation of a novel B1-type cyclin and its quantitative analysis. Plant Mol. Biol. 41: 245-258. 
Trimarchi JM and Lees JA (2002). Sibling rivalry in the E2F family. Nat. Rev. Mol. Cell Biol. 3: 11-20.

Umeda M, Iwamoto N, Umeda-Hara C, Yamaguchi M, et al. (1999). Molecular characterization of mitotic cyclins in rice plants. Mol. Gen. Genet. 262: 230-238.

Vandepoele K, Raes J, De Veylder L, Rouze P, et al. (2002). Genome-wide analysis of core cell cycle genes in Arabidopsis. Plant Cell 14: 903-916.

Vision TJ, Brown DG and Tanksley SD (2000). The origins of genomic duplications in Arabidopsis. Science 290: 2114-2117.

Wang GF, Kong HZ, Sun YJ and Zhang XH (2004). Genome-wide analysis of the cyclin family in Arabidopsis and comparative phylogenetic analysis of plant cyclin-like proteins. Plant Physiol. 135: 1084-1099.

Yamaguchi M, Fabian T, Sauter M, Bhalerao RP, et al. (2000). Activation of CDK-activating kinase is dependent on interaction with H-type cyclins in plants. Plant J. 24: 11-20.

Yu Y, Steinmetz A, Meyer D, Brown S, et al. (2003). The tobacco A-type cyclin, Nicta;CYCA3;2, at the nexus of cell division and differentiation. Plant Cell 15: 2763-2777. 